\title{
INSUFICIÊNCIAS RESPIRATÓRIA E RENAL DESENCADEADAS POR MIOPATIA METABÓLICA POR ACÚMULO DE LIPÍDIOS
}

\author{
Relato de caso
}

\author{
Walther de Oliveira Campos Filho ${ }^{1}$, Edson Nicolini ${ }^{1}$, Maria Auxiliadora Martins ${ }^{1}$, \\ Sérgio Zucoloto², Aníbal Basile Filho ${ }^{3}$
}

\begin{abstract}
RESUMO - Relatamos o caso de um paciente de 26 anos que apresentou quadro clínico de rabdomiólise e mioglobinúria associadas a insuficiência renal e respiratória, três dias após o início de infecção de vias aéreas superiores. Os dados clínicos e laboratoriais, eletroneuromiografia e biópsia muscular conduziram ao diagnóstico de miopatia metabólica por deficiência enzimática relacionada ao metabolismo dos ácidos graxos. 0 paciente evoluiu favoravelmente com a instituição de suporte avançado de vida, incluindo assistência ventilatória e terapia dialítica, adequação dietética e reposição de L-carnitina. Discorremos sobre as diversas miopatias metabólicas, o diagnóstico, o diagnóstico diferencial e o tratamento.
\end{abstract}

PALAVRAS-CHAVE: metabolismo de lipídeos, metabolismo do glicogênio, rabdomiólise, miopatia metabólica, deficiência de carnitina palmitil transferase.

\section{Respiratory and renal dysfunctions due to lipid storage metabolic myopathy: case report}

ABSTRACT - We report a case of a 26-years old male patient with rhabdomyolisis with myoglobinuria associated with acute renal and respiratory failure, that occurred three days after upper airway infection. In the clinical and laboratory investigation of the patient, including electromyography and muscular biopsy, the diagnostic lead to a metabolic myopathy due to an enzymatic deficiency related to a disorder of lipid metabolism. The patient improved successfully with institution of advanced life support, including ventilatory assistence, dialysis, dietary adjust and L-carnitine reposition.

KEY WORDS: lipid metabolism, glycogen metabolism, rhabdomyolysis, myoglobinuria, metabolic myopathy, carnitine palmityl transferase deficiency.

Os lipídios musculares cada vez mais têm recebido atenção devido seu papel como fonte de energia e o conhecimento de seu metabolismo levou à descrição de doenças decorrentes de defeitos na sua utilização ${ }^{1,2}$. Em geral, essas patologias apresentam relação entre miopatia e alteração no metabolismo lipídico. A deficiência enzimática proporciona alteração metabólica em que há limitação na utilização de lipídios como fonte de energia pela célula ${ }^{1,3,4}$. Expressão clínica mais importante desta deficiência são episódios recorrentes de rabdomiólise com mioglobinúria, precipitados principalmente por exercício extenuante ${ }^{1,3-6}$. Entre outras causas que podem desencadear a lise muscular estão a exposição ao frio, privação de sono, jejum prolongado, anestesia e infecções s,8. $^{7}$.

O objetivo deste estudo é relatar o caso de um paciente com deficiência enzimática relacionada ao metabolismo de lipídio diante de situação de alta demanda metabólica, e alertar para um grupo de patologias em que o alto índice de suspeição e tratamento precoce fazem diferença no prognóstico.

\section{CASO}

Um paciente do sexo masculino, 26 anos, branco, foi admitido na Unidade de Emergência do Hospital das Clínicas da Faculdade de Medicina de Ribeirão Preto, apre-

Unidade de Terapia Intensiva do Hospital das Clínicas da Faculdade de Medicina de Ribeirão Preto da Universidade de São Paulo, Ribeirão Preto SP, Brasil (FMRP / USP): ${ }^{1}$ Médico Assistente da Unidade de Terapia Intensiva do Hospital das Clínicas da FMRP / USP; ${ }^{2}$ Professor Titular do Departamento de Cirurgia e Anatomia da FMRP / USP; ${ }^{3}$ Chefe da Disciplina de Terapia Intensiva e Livre-Docente do Departamento de Cirurgia e Anatomia da FMRP / USP.

Recebido 25 Julho 2001, recebido na forma final 15 Março 2002. Aceito 19 Março 2002. 
sentando agitação psicomotora, insuficiência respiratória (38 incursões por minuto), acianótico, pressão arterial de $130 \times 80 \mathrm{mmHg}$, ausculta pulmonar normal, Glasgow 15 pontos, mas com défice motor simétrico, generalizado, computado como grau 3 em 5 pela escala de força motora. O exame da região abdominal e das extremidades não mostrava alterações; orofaringe hiperemiada e sem pontos purulentos. Há 72 horas havia iniciado tratamento para amigdalite purulenta com penicilina benzatina e há 48 horas foi instituído uso empírico de lincomicina.

Como antecedentes pessoais foi relatado que desde a infância apresentava episódios de mialgia e astenia importantes após esforço físico, sempre acompanhadas de hematúria macroscópica. Também apresentava crises convulsivas isoladas.

A análise sanguínea evidenciou creatinina $8,1 \mathrm{mg} / \mathrm{dL}$, uréia $194 \mathrm{mg} / \mathrm{dL}$, sódio $129 \mathrm{mEq} / \mathrm{L}$, potássio 5,6 mEq/L, creatinafosfoquinase $30000 \mathrm{u} / \mathrm{L}$, aspartato aminotransferase $2492 \mathrm{u} / \mathrm{L}$, colinesterase $2128 \mathrm{u} / \mathrm{L}$. A gasometria arterial inicial denotava $\mathrm{PaO} 2$ 88,1 e PCO2 32,4; após 24 horas, $\mathrm{PaO} 2$ 44,8 e a PCO2 53,5. A radiografia não contrastada do tórax e Doppler ecocardiograma não evidenciaram alterações significativas.

Nas 24 horas seguintes à admissão, o paciente apresentou rebaixamento do nível de consciência, piora do padrão respiratório, evoluindo com taquipnéia, hipoxemia e hipercapnia progressivas, denunciando hipoventilação alveolar. A fadiga muscular generalizada se intensificou. Instalou-se quadro de anúria, sendo necessária sua transferência para unidade de terapia intensiva, onde foi submetido a depuração extra renal. A assistência ventilatória mecânica também foi instituída, por incapacidade de se manter volume corrente satisfatório. A relação $\mathrm{PaO} 2 / \mathrm{FiO}_{2}$ logo após a intubação orotraqueal era de 249. Ressaltase, ainda, que não foram utilizados corticosteróides ou bloqueadores neuromusculares durante o período de internação do paciente. Durante a internação o paciente apresentou crises convulsivas que cederam com fenitoína e fenobarbital. A tomografia de crânio e o eletrencefalograma não mostraram alterações.

A urina de coloração âmbar apresentada pelo paciente conduziu a suspeição de hemoglobinúria decorrente de rabdomiólise. Com a hipótese diagnóstica de miopatia metabólica, foi solicitada eletroneuromiografia, que mostrou condução neuromotora preservada e potenciais de unidade motora com duração e amplitude diminuídas, insinuando se tratar de quadro miopático de caráter inflamatório. A biópsia muscular do bíceps esquerdo evidenciou atrofia de algumas fibras musculares do tipo $\mathrm{I}$. $\mathrm{Na}$ coloração pelo oil red observou-se discreto aumento de depósitos vacuolares de lipídios em várias fibras musculares, direcionando o diagnóstico para defeito relacionado ao metabolismo de gorduras, e sugerindo deficiência de carnitina-palmitil-transferase.

Instituiu-se como tratamento de base uma dieta rica em carboidratos e com baixa proporção de lipídios, associada a reposição de L-carnitina por via enteral na dose de
2 gramas a cada hora. A evolução clínica se mostrou favorável após essas medidas, com recuperação da diurese, da força muscular e retirada progressiva da ventilação mecânica, tendo o paciente recebido alta da UTI 19 dias depois, em bom estado geral.

\section{DISCUSSÃO}

As miopatias metabólicas são desordens geneticamente determinadas que acometem a musculatura estriada, em que um simples defeito se associa a um amplo espectro clínico e diferentes defeitos podem gerar manifestações clínicas semelhantes. Essas patologias em geral se manifestam já desde a infância ou durante o período de adolescência ${ }^{2,5,6,9-12}$.

As miopatias associadas a episódios de fraqueza muscular e a mioglobinúria podem estar presentes tanto nas desordens do metabolismo de carboidratos, como no metabolismo de ácidos graxos, nos defeitos da cadeia respiratória celular e na deficiência de mioadenilato deaminase ${ }^{5,13}$ (Tabela 1). Assim, o estudo histoquímico é parte fundamental para o diagnóstico diferencial das miopatias 1,4-6,13. Nas deficiências de enzimas glicolíticas, como a miofosforilase (Doença de Mc Ardle), fosfofrutoquinase (Doença de Tarui), fosfogliceratoquinase, fosfoglicerato mutase e desidrogenase lática, a biópsia muscular evidencia depósitos de glicogênio nas miofibrilas. Quando se trata de defeitos na cadeia respiratória,

Tabela 1. Miopatias indutoras de mioglobinúria recorrente.

Defeitos no metabolismo dos carboidratos

Deficiência de miofosforilase (Doença de Mc Ardle)

Deficiência de fosfofrutoquinase (Doença de Tauri)

Deficiência de fosfogliceratoquinase

Deficiência de fosfoglicerato mutase

Deficiência de lactato desidrogenase

Deficiência de fosforilase $b$ quinase

Defeitos no metabolismo dos lipídios

Deficiência de carnitina palmitil transferase

Deficiência de Acil-CoA desidrogenase de cadeia longa

Deficiência de Acil-CoA desidrogenase de cadeia média

Deficiência de Acil-CoA desidrogenase de cadeia curta

Defeitos na cadeia respiratória celular

Defeitos de complexos I, III, IV

Deficiência de coenzima Q

Deficiência de mioadenilato deaminase 
a histoquímica mostra fibras vermelhas rotas ou desalinhadas. Já nas desordens metabólicas dos ácidos graxos, os depósitos encontrados nas fibras musculares são compostos por lipídios 2,3,5,13,14.

Outra forma de se diferenciar defeitos entre metabolismo de lipídios e carboidratos é a dosagem sérica de lactato e piruvato, coletando o sangue em veia do antebraço, antes e após exercício físico em regime de isquemia do membro superior (prova do manguito). Após o impedimento da circulação, o lactato e o piruvato elevam-se tanto em pessoas normais como em portadores de doenças do metabolismo lipídico. Já nos pacientes com patologias referentes aos carboidratos essa elevação não ocorre ${ }^{13}$.

No caso aqui relatado, o estudo histoquímico evidenciou a presença de vacúolos de lipídios no interior das fibras musculares, descartando a possibilidade de se tratar de patologias do metabolismo de carboidratos e doenças relacionadas à cadeia respiratória celular e direcionando o diagnóstico para as patologias lipídicas (Fig 1).

Basicamente os defeitos na oxidação de ácidos graxos na mitocôndria compreendem defeitos (1) no ciclo da carnitina, representado pela deficiência de carnitina palmitil transferase, e (2) na $\beta$ oxidação das gorduras, em que é mais frequente a deficiência de acil Co-A desidrogenase de cadeia média ${ }^{9,10,13}$.

Nas desordens inatas do metabolismo lipídico, o metabolismo de carboidratos não se altera mas a utilização dos ácidos graxos fica limitada, permane- cendo o paciente todo o tempo num potencial "déficit energético", justamente pela baixa capacidade de oxidação de lipídios ${ }^{711,14-16}$. Nesses casos, qualquer situação de grande demanda metabólica, associada a uma reduzida capacidade de sintetizar ATP e fosfatos de alta energia, culmina em dificuldade de manter a integridade da membrana celular, quando ocorre a citólise e se deflagra a mioglobinúria. Portanto, a mioglobinúria decorrente de distúrbios metabólicos tem como denominador comum a insuficiente utilização do substrato para o metabolismo energético ou uma crítica deficiência de substrato diante de uma demanda energética excessiva.

Durante o exercício físico, a fonte de energia muscular provém inicialmente do metabolismo de carboidratos. Se mantida a atividade muscular, gradualmente os lipídios tornam-se a principal fonte energética, já que a reserva de glicogênio começa a se esgotar em torno de 40 minutos após iniciado o esforço físico ${ }^{2,7,11}$. Depois de findada a reserva glicídica, o organismo lança mão dos ácidos graxos para obtenção de energia suficiente para suprir tal demanda, e a oxidação dessas gorduras se dá no interior das mitocrôndrias. Para que os lipídios alcancem o interior da organela para a geração de energia, eles precisam ser carreados por uma série de enzimas, em que se destaca a carnitina palmitil transferase (CPT). Essa enzima, que se apresenta nas faces externa e interna da membrana interna da mitocrôndria (CPT tipos 1 e 2, respectivamente), é que transpassa os ácidos graxos para o interior dessa organela e propicia sua oxidação $0^{3,17}$.

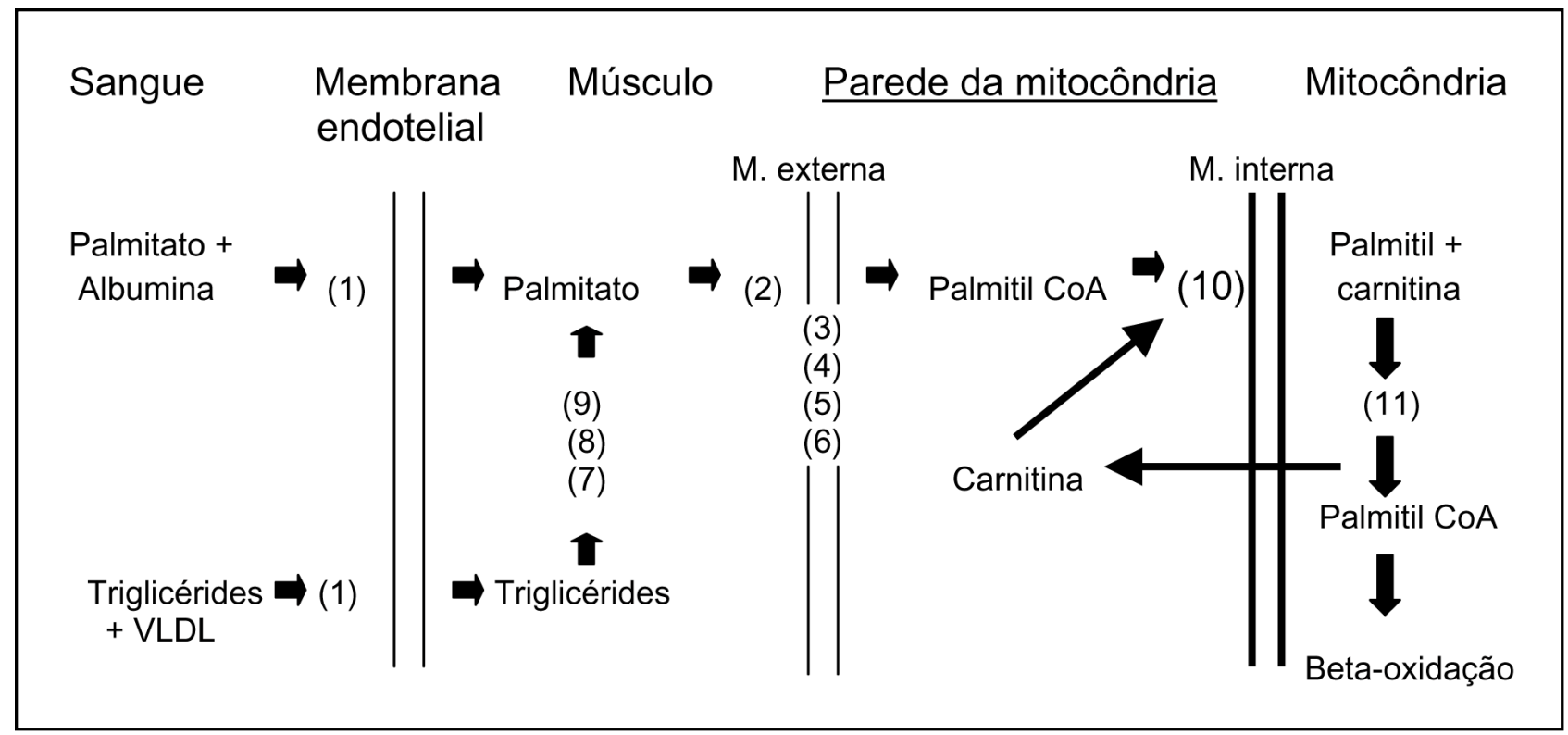

Fig 1. Esquema da via metabólica dos ácidos graxos triglicérides para entrarem no ciclo de krebs: (1) Lipases; (2) Palmitil Coa sintetase; (3) Glicerol-I-fosfato; (4) Glicerol-fosfato acil-transferase; (5) Fosfatidato fosfatase; (6) Diglicérido acil-transferase; (7) Triglicérido lipase; (8) Diglicérido lipase; (9) Monoglicérido lipase; (10) Carnitina-palmitil-transferase I; (11) Carnitina-palmitil-transferase II. 
Em pacientes com miopatias metabólicas com deficiência enzimática, o esforço muscular continuado induz a dor, a edema e a fraqueza muscular, refletindo a necrose do músculo, representada por rabdomiólise e mioglobinúria ${ }^{5,6,13}$.

A fraqueza muscular pode alcançar grandes proporções quando o paciente pode apresentar insuficiência ventilatória ${ }^{6,8}$. A rabdomiólise acentuada pode proporcionar depósito de mioglobina nos túbulos renais e levar a necrose tubular aguda, desencadeando, assim, a insuficiência renal aguda. Se não forem terapeuticamente conduzidas corretamente e de forma urgente, essas duas complicações podem evoluir para o óbito $7,8,11,17,18$.

Essas patologias também geram, com grande frequência, depleção secundária de carnitina, justificando a reposição dessa substância como parte do tratamento $1,2,4,9,10$. A base desse tratamento é oferecer uma dieta rica em carboidratos e com baixo teor de gorduras. Quando associadas a dieta e a reposição de carnitina, a recuperação pode se dar de forma mais rápida nesses pacientes ${ }^{1,2}$, fato que pode ser corroborado pelo caso relatado.

A biópsia também pode ser analisada do ponto de vista estrutural da fibra muscular. As fibras musculares do tipo I, ou fibras vermelhas, dependem, quase que exclusivamente, da oxidação dos ácidos graxos para suprir seu requerimento energético e, portanto, são as fibras onde se encontra com maior frequência tais alterações, dentre as quais se destaca a atrofia ${ }^{1,4}$. A biópsia muscular do caso relatado evidenciou atrofia de fibras musculares do tipo I, semelhante aos relatos iniciais de DiMauro e Scarlato, quando apresentaram casos de deficiência de CPT 1,3,4.

Outra característica dessas patologias é a não produção ou produção diminuída e tardia de corpos cetônicos durante o jejum. ${ }^{7,11,12,15}$. Também, os exames laboratoriais, a eletroneuromiografia, e a biópsia muscular podem não apresentar alterações nos períodos de acalmia ${ }^{1,3,4,11,15,16}$. O diagnóstico definitivo é selado através das dosagens séricas, e do estudo histoquímico e bioquímico de homogenados musculares, quantificando as atividades enzimáticas $13,4,6,11,14,17$, o que não foi possível de se realizar com esse paciente.
Embora, no caso em questão, não tenha sido detectada de forma específica a enzima deficiente, 0 diagnóstico de deficiência enzimática pode ser estabelecido, já que há muitas evidências de se tratar de defeito metabólico relacionado ao metabolismo de ácidos graxos. É importante ressaltar que a suspeição e abordagem precoces, no âmbito das unidades de terapia intensiva, tem fundamental importância no sucesso terapêutico e, portanto, no prognóstico desses pacientes.

\section{REFERÊNCIAS}

1. Bank WJ, DiMauro S, Bonilla E, Capuzzi D M, Rowland L P. A disorder of muscle lipid metabolism and myoglobinuria: absence of carnitine palmityl transferase. N Engl J Med 1975;292:443-448.

2. DiMauro S, DiMauro PMM. Muscle carnitine palmityl transferase deficiency and myoglobinuria. Science 1983;182:929.

3. Engel AG. Doenças do músculo e da junção neuromuscular. In Bennet JC, Plum F. Tratado de medicina interna. 20.Ed. Rio de Janeiro: Guanabara Koogan, 1997:2380-2397.

4. Scarlato G, Albizatti MG, Bassi S, Cerri C, Frattola L. A case os lipid storage myopathy with carnitine deficiency: biochemical and eletromyographic correlations. Eur Neurol 1977;16:222-229.

5. Rifai Z, Griggs RC. Metabolic myopathies. In: Samuels MA, Feske S. Office practice of neurology. New York: Churchill Livingstone, 1996: 600-604.

6. Rowland LP. Myoglobinúria. In Rowland LP. Merrit's textbook of neurology. 9.Ed. New York: Williams \& Wilkins, 1995;788-790.

7. Bertorini T, Yeh YY, Trevisan C, Stadlan E, Sabesin S, DiMauro S. Carnitine palmityl transferase deficiency: myoglobinuria and respiratory failure. Neurology 1980;30:263-271.

8. Katsuya H, Misumi M, Ohtani Y, Miike T. Postanesthesic acute renal failure due to carnitine pamityl transferase deficiency. Anesthesiology 1988;68:945-948.

9. Lyon G, Adams RD, Kolodny EH. The neurology of neonatal hereditary metabolic diseases. In Lyon G. Neurology of hereditary metabolic diseases of children. 2.Ed. New York: Mc Graw-Hill, 1996;6-44.

10. Lyon G, Adams RD, Kolodny EH. Early infantile progressive metabolic encefalopathies: clinical problems and diagnostic considerations. In Lyon G, Neurology of hereditary metabolic diseases of children. 2.Ed. New York, Mc Graw-Hill, 1996:44-123.

11. Werneck LC, Bôer CA, Papadimitriou A, DiMauro S. Myopathy due to carnitine palmitoyltransferase: report of 2 cases with enzymatic analyses on muscle tissue. Arq Neuropsiquiatr 1983;41:377-384.

12. Werneck LC. Miopatias metabólicas. In Melo-Souza SE. Tratamento das doenças neurológicas. Rio de Janeiro: Guanabara Koogan, 2000:553-556.

13. DeVivo DC. Mitochondrial diseases with mutations of nuclear DNA. Mitochondrial encephalomyopathies. In Rowland LP. Merritt's textbook of neurology. 9.Ed. New York: Williams \& Wilkins, 1995;626-629.

14. Angelini C, Freddo L, Battistella P, et al. Carnitine palmityl transferase deficiency: clinical variability, carrier detection, and autosomal-recessive inheritance. Neurology 198;131:883-886.

15. Layzer RB, Lewis SF. Clinical disorders of muscle energy metabolism. Med Sci Sports Exerc 1984;16:451-455.

16. Mongini T, Doriguzzi C, Palmucci L, Chiado-Piat L, Manilcalco M, Schiffer D. Myoglobinuria and carnitine palmityl transferase deficiency in father and son. J Neurol 1991;238:323-324.

17. Brownell AK, Severson DL, Thompson CD, Fletcher T. Cold induced rhabdomyolysis in carnitine palmityl transferase deficiency. Can J Neurol Sci 1979;6:367-370.

18. Kelly KJ, Garland JS, Tang TT, Shug AL, Chusid MJ. Fatal rhabdomyolysis following influenza infection in a girl with familial carnitine palmityl transferase. Pediatrics 1989;84:312-316. 